\title{
Demande de sédation continue jusqu'au décès : la place des soins psychiques
}

\section{Les recommandations de la SFPO}

\section{Request for Continuous Sedation Until Death: the Place of Mental Care}

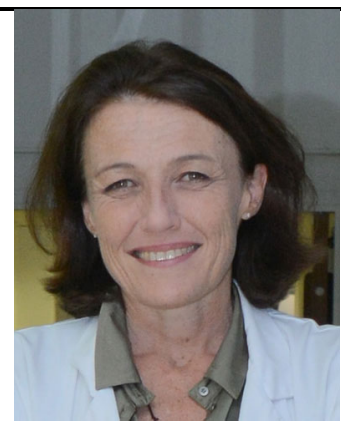

\section{SFPO Guidelines}

\author{
S. Dauchy et le Conseil d'Administration de la Société Française de Psycho-Oncologie* \\ (C) Lavoisier SAS 2019
}

L'accès à la sédation profonde et continue jusqu'au décès (SPCJD), chez un patient atteint de maladie grave et incurable dont le pronostic vital est engagé à court terme et qui présente une souffrance réfractaire, est un droit reconnu par la loi Claeys-Leonetti [1]. Dans la pratique, prendre en compte cette demande ne dispense en rien le clinicien, d'une part, de l'analyse des causes de la souffrance qui sous-tend cette demande (puisque son caractère réfractaire implique d'avoir mobilisé pour la traiter des ressources adaptées) ; et d'autre part, de la prise en compte de l'intentionnalité sousjacente à la demande de sédation. La Société Française et Francophone de Psycho-Oncologie propose ici quelques pistes de réflexion pour soutenir l'analyse clinique de ces demandes et aider les cliniciens, et parmi eux les professionnels de soins psychiques, à y répondre au mieux dans le respect de l'autonomie des patients.

\section{Souffrance réfractaire}

La souffrance est une notion globale, particulièrement en fin de vie (voir la définition de la total pain par Cicely Saunders [2]). Elle doit être envisagée dans une perspective

\section{S. Dauchy $(\bowtie)$}

Département interdisciplinaire de soins de support, unité de psycho-oncologie Gustave-Roussy, université Paris-Saclay, 114, rue Édouard-Vaillant, F-94805 Villejuif, France

e-mail : sarah.dauchy@gustaveroussy.fr

*Conseil d'administration de la Société Française de PsychoOncologie (SFPO) :

M.-F. Bacqué · N. Bendrihen · B. Bernard · H. de la Menardière

M. Derzelle · S. Dolbeault · F. Ellien · J. Guérin · C. Glineur ·

G. Ganem · P. Guex · L. Lemaitre - C. Lemogne · A. Lesieur ·

Y. Libert · E. Marx · J.-F. Morere · N. Pelicier · A. Polomeni ·

N. Porée $\cdot$ M. Reich $\cdot$ C. Segura $\cdot$ E. Seigneur multidimensionnelle où les aspects physiques, psychologiques et existentiels sont intriqués [3]. Y contribuent les symptômes physiques bien sûr (douleur, dyspnée...) ou psychiques (ex. : angoisse), mais aussi l'appréciation subjective par le patient de sa situation (information, représentations, respect des valeurs, perte de repères, dignité et estime de soi...), ainsi qu'une dimension sociale et relationnelle (relations avec l'entourage, avec l'équipe soignante, vécu d'abandon, deuil anticipé, rupture du cadre habituel...).

On ne peut donc pas opposer d'un côté souffrance physique et souffrance psychologique, souffrances qui pourraient relever de soins respectivement physiques ou psychiques, et de l'autre souffrance existentielle, qui implicitement renverrait plus à un système de pensée et de valeurs propre à celui qui l'expérimente et s'avérerait insensible à un projet de soins. Les travaux de Kissane [4], de Chochinov et al. [5], de Grassi [6] et de Robinson [7] sur la démoralisation et les ressources thérapeutiques des psychothérapies existentielles, narratives, « de la dignité » viennent illustrer comment ces dimensions sont interconnectées et comment la souffrance existentielle peut être au moins partiellement sensible à un projet thérapeutique ou à une prise en charge, en particulier lorsque ceux-ci mobilisent le support relationnel, les conditions de vie, le respect des droits du patient et son autonomie, autonomie qui ne peut être réduite à la seule possibilité de demander une sédation [8].

L'évaluation de la souffrance en fin de vie implique donc une évaluation fine de l'état émotionnel et de l'état cognitif, ainsi qu'une appréciation de la dynamique psychosociale [9]. On pourra parler de souffrance réfractaire lorsqu'après évaluation de ses causes les mesures thérapeutiques potentiellement efficaces auront été mises en œuvre (traitement des symptômes physiques et émotionnels, mais aussi lorsque cela est possible restauration des repères, de la dignité, lutte contre la perte de sens...). 
Si le patient reste le seul capable d'apprécier le caractère intolérable de sa propre souffrance, l'identifier comme réfractaire implique une démarche multidisciplinaire où le clinicien doit faire la preuve de la mobilisation des moyens nécessaires tant à l'évaluation qu'à la proposition de soins qui peut être faite au patient. L'implication dans la réflexion multidisciplinaire d'un professionnel de soins psychiques formé aux spécificités de la fin de vie est dans ce contexte très largement recommandée.

\section{Intentionnalité de la demande}

Prendre en compte l'intentionnalité sous-jacente à la demande de SPCJD, sédation qui a pour effet d'altérer profondément et de façon définitive la conscience, implique par ailleurs de considérer la possible existence sous-jacente d'un désir de hâter la mort.

Il existe en effet une littérature internationale abondante sur ce désir de hâter la mort (Wish to Hasten Death), dont une définition consensuelle est proposée comme une réaction à la souffrance, dans un contexte d'engagement du pronostic vital, où le patient ne voit d'autre porte de sortie que d'accélérer sa mort. Ce souhait peut s'exprimer spontanément ou lors d'un échange où la question est posée. Il doit être distingué de l'acceptation d'une mort proche ou d'un souhait de mourir naturellement même le plus tôt possible. Le désir de hâter la mort peut surgir en réponse à un ou plusieurs facteurs : des symptômes physiques (présents ou attendus), une détresse psychologique (dépression, désespoir, peurs, etc.), une souffrance existentielle (par exemple perte de sens de la vie) ou sociale (sentiment d'être un fardeau...) [10].

Les données issues des recherches sur ce désir de hâter la mort [11] doivent être prises en compte pour les raisons suivantes :

- il a un caractère potentiellement dynamique et évolutif [12];

- il peut être défini comme une réponse à une situation donnée, voire comme un moyen d'adaptation, par exemple pour faire face à l'incertitude d'une agonie, à la peur de la souffrance [13]; comme tout moyen adaptatif, il peut donc évoluer si les ressources du patient ou la situation à laquelle il fait face évoluent. Il a ainsi été montré que le WTHD peut évoluer si les symptômes physiques s'améliorent ou si la symptomatologie dépressive diminue [14-16];

- il a également été montré que l'état psychologique d'un patient (dépression, démoralisation ou perte d'espoir, perte de contrôle, faible estime de soi) est le médiateur de l'association entre le désir de hâter la mort et le degré de souffrance perçue du patient, ou sa qualité de vie $[17,7]$.
On sera donc vigilant devant une demande de SPCJD à intégrer l'évaluation de celle-ci dans un contexte dynamique et à respecter un délai suffisant pour prendre en compte ce caractère potentiellement évolutif le temps de mettre en jeu les ressources thérapeutiques adaptées, si elles sont nécessaires et possibles. Les propositions thérapeutiques doivent, comme toute prise en charge complexe, s'intégrer dans un réel echange pluridisciplinaire, garant $d u$ respect de l'éthique.

Le risque, sinon, est celui de la perte de chance, voire de la non-assistance à personne en danger par mobilisation insuffisante de ressources thérapeutiques et de prise en charge adéquates.

Il sera également nécessaire, dans cette situation, de fournir au patient une information claire et loyale sur l'évolution possible de son appréhension de la situation.

\section{Remarques complémentaires}

\section{Sédation transitoire ou SPCJD?}

La sédation transitoire reste une des propositions thérapeutiques possibles devant une souffrance psychique ou existentielle potentiellement réfractaire ; elle rend néanmoins impossible toute approche psychothérapeutique ou relationnelle; elle ne remplace pas la SPCJD, qui reste un droit inaliénable du patient - mais le respect des règles éthiques imposant de tenir compte de la complexité de l'évaluation clinique de la demande peut dans un premier temps une sédation transitoire.

\section{Que faire en cas d'atteinte du discernement ?}

La loi ne prévoit pas la situation d'atteinte du discernement, qui d'un point de vue légal ne prive pas le patient de ses droits civiques mais qui contraint pour des raisons éthiques à prendre en compte avec la plus grande prudence ses demandes, sous peine d'une perte de chances. C'est le cas par exemple pour le patient atteint de maladie psychiatrique grave et décompensée, de troubles cognitifs majeurs ou d'une déficience mentale profonde. La prise en compte d'éventuelles directives anticipées et celle de l'avis de la personne de confiance lorsqu'il y en a une seront ici fondamentales.

\section{Références}

1. Claeys-Leonetti LOI n ${ }^{\circ} 2016-87$ du 2 février 2016 créant de nouveaux droits en faveur des malades et des personnes en fin de vie JORF $\mathrm{N}^{\circ} 0028$ du 3 février 2016. NOR: AFSX1507642L ELI: https://www.legifrance.gouv.fr/eli/loi/2016/2/2/AFSX1507 642L/jo/texte - Alias: https://www.legifrance.gouv.fr/eli/loi/2016/ 2/2/2016-87/jo/texte 
2. Clark D (1999) Total pain', disciplinary power and the body in the work of Cicely Saunders, 1958-1967. Soc Sci Med 49:727-36

3. Sainton J, Derzelle M (2019) Sédation palliative et sédation terminale. Médecine Palliative, Elsevier Masson, Paris

4. Kissane DW (2012) The relief of existential suffering. Arch Intern Med 172:1501-5

5. Chochinov HM, Hack T, McClement S, et al (2002) Dignity in the terminally ill: a developing empirical model. Soc Sci Med 54:433-43

6. Grassi L et Nanni MG (2016) Demoralization syndrome: new insights in psychosocial cancer care. Cancer 122: 2130-33

7. Robinson S, Kissane DW, Brooker J, et al (2017) The relationship between poor quality of life and desire to hasten death: a multiple mediation model examining the contributions of depression, demoralization, loss of control, and low self-worth. J Pain Symptom Manage 53:243-9

8. Galushko M, Frerich G, Perrar KM, et al (2016) Desire for hastened death: how do professionals in specialized palliative care react? Psycho-Oncology 25:536-43

9. Derzelle M (2007) Clinique de la douleur chronique et approche psychanalytique. RFPO 2:88-92

10. Balaguer A, Monforte-Royo C, Porta-Sales J, et al (2016) An international consensus definition of the wish to hasten death and its related factors. PLoS One 11:e0146184

11. Rodríguez-Prat A, Balaguer A, Booth A, et al (2017) Understanding patients' experiences of the wish to hasten death: an updated and expanded systematic review and meta-ethnography. BMJ Open 7:e016659. doi: 10.1136/bmjopen-2017-016659
12. Ohnsorge K, Gudat H, Rehmann-Sutter C (2014) Intentions in wishes to die: analysis and a typology - a report of 30 qualitative case studies of terminally ill cancer patients in palliative care. Psycho-Oncology 23:1021-6

13. Pestinger M, Stiel S, Elsner F, et al (2015) The desire to hasten death: using Grounded Theory for a better understanding "When perception of time tends to be a slippery slope". Palliat Med 29:711-9

14. Price A, Lee W, Goodwin L, et al (2011) Prevalence, course and associations of desire for hastened death in a UK palliative population: a cross-sectional study. BMJ Support Palliat Care 1:140-8

15. O'Mahony S, Goulet J, Kornblith A, et al (2005) Desire for hastened death, cancer pain and depression: report of a longitudinal observational study. J Pain Symptom Manage 29:446-57

16. Breitbart W, Rosenfeld B, Pessin H, et al (2000) Depression, hopelessness, and desire for hastened death in terminally ill patients with cancer. JAMA 284:2907-11

17. Bacqué MF (2002) Dépression en fin de vie : deuil de soi ou détresse existentielle ? Calvaire 2000. Revue française des unités de soins palliatifs $12: 21-8$

18. Guerrero-Torrelles M, Monforte-Royo C, Tomás-Sábado J, et al (2017) Meaning in life as a mediator between physical impairment and the wish to hasten death in patients with advanced cancer. J Pain Symptom Manage 54:826-34

19. Rodin G, Zimmermann C, Rydall A, et al (2007) The desire for hastened death in patients with metastatic cancer. J Pain Symptom Manage 33:661-75 